\title{
Wigner distribution functions for complex dynamical systems: The emergence of the Wigner-Boltzmann equation
}

\author{
Dries Sels* and Fons Brosens ${ }^{\dagger}$ \\ Physics Department, University of Antwerp, Universiteitsplein 1, 2060 Antwerpen, Belgium
}

(Received 31 July 2013; published 1 October 2013)

\begin{abstract}
The equation of motion for the reduced Wigner function of a system coupled to an external quantum system is presented for the specific case when the external quantum system can be modeled as a set of harmonic oscillators. The result is derived from the Wigner function formulation of the Feynman-Vernon influence functional theory. It is shown how the true self-energy for the equation of motion is connected with the influence functional for the path integral. Explicit expressions are derived in terms of the bare Wigner propagator. Finally, we show under which approximations the resulting equation of motion reduces to the Wigner-Boltzmann equation.
\end{abstract}

DOI: 10.1103/PhysRevE.88.042101

PACS number(s): 05.30.-d, 67.10.Hk

\section{INTRODUCTION}

Recently, we derived the propagator for the reduced Wigner function of a system coupled to an external quantum system [1]. The resulting path integral can only be solved exactly for a limited set of problems. An example of such a system is the Caldeira-Leggett model [2] in which the external quantum system consists of a set of independent harmonic oscillators bilinearly coupled to the system of interest. As shown in [1], the resulting time evolution of the system can be modeled by a stochastic differential equation subject to correlated noise. In the Ohmic case, the noise is white and the time evolution of the system is of the Wigner-Fokker-Planck type, a detailed discussion of which can be found in [3]. In this work, we derive the equation of motion for the reduced Wigner function for a system coupled to a set of independent oscillators in a slightly more complicated way. We will still consider the coupling to be linear in the oscillator coordinate but nonlinear in the system coordinate. It is the linearity in the oscillator coordinate that enables us to eliminate them, but the nonlinearity in the system coordinate makes it impossible to solve the resulting path integral using standard techniques. Note that this situation is quite common as, in the language of quantum field theory, it describes a particle coupled to a gauge field.

The main result of this work can be summarized as follows. Consider a system and an environment described by the following Hamiltonian:

$$
\begin{aligned}
H= & \frac{\mathbf{p}^{2}}{2 m}+V(\mathbf{x}, t)+\sum_{k} \hbar \omega_{k}\left(b_{k}^{\dagger} b_{k}+\frac{1}{2}\right) \\
& +\sum_{k}\left[\gamma(\mathbf{k}) \exp (-i \mathbf{k} \cdot \mathbf{x}) b_{k}^{\dagger}+\gamma^{*}(\mathbf{k}) \exp (i \mathbf{k} \cdot \mathbf{x}) b_{k}\right] .
\end{aligned}
$$

Assume furthermore that the phonon bath is initially in thermal equilibrium, but leave the initial density matrix for the particle unspecified. We shall show that the Liouville equation for the

\footnotetext{
*Author to whom all correspondence should be addressed: dries.sels@uantwerpen.be

†fons.brosens@uantwerpen.be
}

reduced Wigner function of the system is then given by

$$
\begin{aligned}
& \left(\frac{\partial}{\partial t}+\frac{\mathbf{p}}{m} \cdot \nabla\right) f(\mathbf{x}, \mathbf{p}, t) \\
& =\int d \mathbf{p}^{\prime} \Lambda\left(\mathbf{x}, \mathbf{p}-\mathbf{p}^{\prime}, t\right) f\left(\mathbf{x}, \mathbf{p}^{\prime}, t\right) \\
& \quad+\iiint d t^{\prime} d \mathbf{x}^{\prime} d \mathbf{p}^{\prime} \Sigma_{R}\left(\mathbf{x}, \mathbf{p}, t \mid \mathbf{x}^{\prime}, \mathbf{p}^{\prime}, t^{\prime}\right) f\left(\mathbf{x}^{\prime}, \mathbf{p}^{\prime}, t^{\prime}\right),
\end{aligned}
$$

where a retarded self-interaction $\Sigma_{R}$ and a Wigner kernel $\Lambda$ from the external potential are given by

$$
\begin{aligned}
\Sigma_{R}\left(\mathbf{x}, \mathbf{p}, t \mid \mathbf{x}^{\prime}, \mathbf{p}^{\prime}, t^{\prime}\right) & \\
= & \Theta\left(t-t^{\prime}\right) \sum_{k} \frac{2|\gamma(\mathbf{k})|^{2}}{\hbar^{2}}\left[n _ { B } ( \omega _ { k } ) \operatorname { c o s } \left[\mathbf{k} \cdot\left(\mathbf{x}-\mathbf{x}^{\prime}\right)\right.\right. \\
& \left.\left.-\omega_{k}\left(t-t^{\prime}\right)\right]+\left[n_{B}\left(\omega_{k}\right)+1\right] \cos \left[\mathbf{k} \cdot\left(\mathbf{x}-\mathbf{x}^{\prime}\right)+\omega_{k}\left(t-t^{\prime}\right)\right]\right] \\
& \times\left[K_{0}\left(\mathbf{x}, \mathbf{p}-\frac{\hbar \mathbf{k}}{2}, t \mid \mathbf{x}^{\prime}, \mathbf{p}^{\prime}+\frac{\hbar \mathbf{k}}{2}, t^{\prime}\right)\right. \\
- & \left.K_{0}\left(\mathbf{x}, \mathbf{p}+\frac{\hbar \mathbf{k}}{2}, t \mid \mathbf{x}^{\prime}, \mathbf{p}^{\prime}+\frac{\hbar \mathbf{k}}{2}, t^{\prime}\right)\right], \\
& \Lambda(\mathbf{x}, \mathbf{p}, t)=-\frac{i}{\hbar} \int\left[V\left(\mathbf{x}+\frac{\boldsymbol{\xi}}{2}, t\right)-V\left(\mathbf{x}-\frac{\boldsymbol{\xi}}{2}, t\right)\right] \\
& \times \exp \left(-\frac{i}{\hbar} \boldsymbol{\xi} \cdot \mathbf{p}\right) \frac{d \boldsymbol{\xi}}{(2 \pi \hbar)^{3}} .
\end{aligned}
$$

The complete influence of the bath on the system of interest is contained in the retarded self-energy $\Sigma_{R}$. In expression (3), $n_{B}$ denotes the Bose-Einstein distribution at zero chemical potential and $K_{0}$ denotes the bare Wigner propagator (of the system without the bath). Most of this manuscript is concerned with the derivation of (2) and it is organized in the following way. First, we will derive a perturbation series for reduced Wigner function propagators. By resumming this series exactly, we find a Dyson integral equation for the reduced propagator. The equation of motion for the reduced Wigner function can then simply be derived from this integral equation. This will result in an explicit expression for the retarded self-energy in terms of the bath properties contained in the influence functional and the bare propagator of the system. The remaining part of this work will be devoted to a detailed discussion of the result and its application to translational invariant systems. It will be shown how the self-energy 
simplifies to the same expression as the one predicted by the celebrated Fermi's golden rule under the usual assumptions of weak coupling and linear response.

\section{PERTURBATION THEORY}

Consider the following propagator:

$$
\begin{aligned}
& K_{w}\left(\mathbf{x}_{b}, \mathbf{p}_{b}, t_{b} \mid \mathbf{x}_{a}, \mathbf{p}_{a}, t_{a}\right) \\
&=\frac{1}{(2 \pi \hbar)^{3}} \int \begin{array}{c}
x\left(t_{b}\right)=x_{b} \\
p\left(t_{b}\right)=m \dot{x}_{b} \\
x\left(t_{a}\right)=x_{a} \\
p\left(t_{a}\right)=m \dot{x}_{a}
\end{array} \\
& \quad \times \operatorname{Dxp}\left(-\frac{i}{\hbar} S_{0}[\mathbf{x}, \boldsymbol{\xi}]+\frac{i}{\hbar} \Phi[\mathbf{x}+\boldsymbol{\xi} / 2, \mathbf{x}-\boldsymbol{\xi} / 2]\right),
\end{aligned}
$$

where $S_{0}[\mathbf{x}, \boldsymbol{\xi}]$ is the bare action associated with Hamiltonian (1),

$$
S_{0}[\mathbf{x}, \boldsymbol{\xi}]=\int_{t_{a}}^{t_{b}} m \ddot{\mathbf{x}}(t) \cdot \boldsymbol{\xi}(t)+\Delta(\mathbf{x}(t), \boldsymbol{\xi}(t), t) d t,
$$

with $\Delta(\mathbf{x}(t), \boldsymbol{\xi}(t), t)=V\left(\mathbf{x}+\frac{\xi}{2}, t\right)-V\left(\mathbf{x}-\frac{\xi}{2}, t\right)$,

and where $\Phi$ is the influence phase caused by the interactions with the bath. It was shown in [1] that, if the bath was initially in thermal equilibrium, these influence phases are of the form

$\frac{i}{\hbar} \Phi[\mathbf{x}+\boldsymbol{\xi} / 2, \mathbf{x}-\boldsymbol{\xi} / 2]=\int_{t_{a}}^{t_{b}} \int_{t_{a}}^{\tau_{2}} g\left(\mathbf{x}_{\tau_{1}}, \mathbf{x}_{\tau_{2}}, \boldsymbol{\xi}_{\tau_{1}}, \boldsymbol{\xi}_{\tau_{2}}\right) d \tau_{1} d \tau_{2}$, where the subindex indicates the time at which the variables must be evaluated. A short derivation of the influence functional for Hamiltonian (1) will be given in the next section. Clearly, the above path integral is difficult to solve as $g$ is in general a nonlinear function of both $\mathbf{x}$ and $\xi$ and it contains nonlocal terms in time. Hence we proceed by expanding the propagator in a Taylor series around $g=0$,

$$
\begin{aligned}
K_{w}\left(\mathbf{x}_{b}, \mathbf{p}_{b}, t_{b} \mid \mathbf{x}_{a}, \mathbf{p}_{a}, t_{a}\right) \\
=\frac{1}{(2 \pi \hbar)^{3}} \int_{\begin{array}{c}
x\left(t_{b}\right)=x_{b} \\
p\left(t_{b}\right)=m \dot{x}_{b} \\
x\left(t_{a}\right)=x_{a} \\
p\left(t_{a}\right)=m \dot{x}_{a}
\end{array}} \mathcal{D} \mathbf{x} \int \mathcal{D} \boldsymbol{\xi} \exp \left(-\frac{i}{\hbar} S_{0}[\mathbf{x}, \boldsymbol{\xi}]\right) \\
\quad \times \sum_{n} \frac{\left(\int_{t_{a}}^{t_{b}} \int_{t_{a}}^{\tau_{2}} g\left(\mathbf{x}_{\tau_{1}}, \mathbf{x}_{\tau_{2}}, \boldsymbol{\xi}_{\tau_{1}}, \boldsymbol{\xi}_{\tau_{2}}\right) \mathrm{d} \tau_{1} \mathrm{~d} \tau_{2}\right)^{n}}{n !} .
\end{aligned}
$$

Consider now in more detail the expression for the first-order correction, $n=1$,

$$
\begin{aligned}
& K_{1}\left(\mathbf{x}_{b}, \mathbf{p}_{b}, t_{b} \mid \mathbf{x}_{a}, \mathbf{p}_{a}, t_{a}\right) \\
& =\int_{t_{a}}^{t_{b}} d \tau_{2} \int_{t_{a}}^{\tau_{2}} d \tau_{1} \frac{1}{(2 \pi \hbar)^{3}} \int_{\substack{x\left(t_{b}\right)=x_{b} \\
p\left(t_{b}\right)=m \dot{x}_{b} \\
x\left(t_{a}\right)=x_{a} \\
p\left(t_{a}\right)=m \dot{x}_{a}}} \mathcal{D} \mathbf{x} \\
& \quad \times \int \mathcal{D} \boldsymbol{\xi} \exp \left(-\frac{i}{\hbar} S_{0}[\mathbf{x}, \boldsymbol{\xi}]\right) g\left(\mathbf{x}_{\tau_{1}}, \mathbf{x}_{\tau_{2}}, \boldsymbol{\xi}_{\tau_{1}}, \boldsymbol{\xi}_{\tau_{2}}\right) .
\end{aligned}
$$

Now we use the Fourier representation of $g$, i.e.,

$$
\begin{aligned}
& g\left(\mathbf{x}_{\tau_{1}}, \mathbf{x}_{\tau_{2}}, \boldsymbol{\xi}_{\tau_{1}}, \boldsymbol{\xi}_{\tau_{2}}\right) \\
& \quad=\iint g^{\prime}\left(\mathbf{x}_{\tau_{1}}, \mathbf{x}_{\tau_{2}}, \mathbf{p}, \mathbf{p}^{\prime}\right) \exp \left(\frac{i}{\hbar}\left(\mathbf{p} \cdot \boldsymbol{\xi}_{\tau_{1}}+\mathbf{p}^{\prime} \cdot \boldsymbol{\xi}_{\tau_{2}}\right)\right) d \mathbf{p} d \mathbf{p}^{\prime},
\end{aligned}
$$

to arrive at the following expression for $K_{1}$ :

$$
\begin{aligned}
K_{1}\left(\mathbf{x}_{b}, \mathbf{p}_{b}, t_{b} \mid \mathbf{x}_{a}, \mathbf{p}_{a}, t_{a}\right)= & \int_{t_{a}}^{t_{b}} d \tau_{2} \int_{t_{a}}^{\tau_{2}} d \tau_{1} \iint d \mathbf{p} d \mathbf{p}^{\prime} \frac{1}{(2 \pi \hbar)^{3}} \int_{\substack{x\left(t_{b}\right)=x_{b} \\
p\left(t_{b}\right)=m \dot{x}_{b} \\
x\left(t_{a}\right)=x_{a}}} \mathcal{D} \mathbf{x} \int \mathcal{D} \xi g^{\prime}\left(\mathbf{x}_{\tau_{1}}, \mathbf{x}_{\tau_{2}}, \mathbf{p}, \mathbf{p}^{\prime}\right) \\
& \times \exp \left[-\frac{i}{\hbar}\left(S_{0}[\mathbf{x}, \boldsymbol{\xi}]-\int_{t_{a}}^{t_{b}}\left[\mathbf{p} \delta\left(t-\tau_{1}\right)+\mathbf{p}^{\prime} \delta\left(t-\tau_{2}\right)\right] \cdot \boldsymbol{\xi}_{t} d t\right)\right]
\end{aligned}
$$

Clearly the additional terms in the action will cause momentum jumps of size $\mathbf{p}$ and $\mathbf{p}^{\prime}$ at times $\tau_{1}$ and $\tau_{2}$, respectively. If we furthermore make use of the fact that the zeroth-order propagator satisfies the Chapman-Kolmogorov equation or chain rule, then we directly arrive at

$$
\begin{aligned}
K_{1}\left(\mathbf{x}_{b}, \mathbf{p}_{b}, t_{b} \mid \mathbf{x}_{a}, \mathbf{p}_{a}, t_{a}\right)= & \int_{t_{a}}^{t_{b}} d \tau_{2} \int_{t_{a}}^{\tau_{2}} d \tau_{1} \iint d \mathbf{p} d \mathbf{p}^{\prime} \iint d \mathbf{x}_{2} d \mathbf{p}_{2} \iint d \mathbf{x}_{1} d \mathbf{p}_{1} g^{\prime}\left(\mathbf{x}_{1}, \mathbf{x}_{2}, \mathbf{p}, \mathbf{p}^{\prime}\right) \\
& \times K_{0}\left(\mathbf{x}_{b}, \mathbf{p}_{b}, t_{b} \mid \mathbf{x}_{2}, \mathbf{p}_{2}, \tau_{2}\right) K_{0}\left(\mathbf{x}_{2}, \mathbf{p}_{2}-\mathbf{p}^{\prime}, \tau_{2} \mid \mathbf{x}_{1}, \mathbf{p}_{1}+\mathbf{p}, \tau_{1}\right) K_{0}\left(\mathbf{x}_{1}, \mathbf{p}_{1}, \tau_{1} \mid \mathbf{x}_{a}, \mathbf{p}_{a}, t_{a}\right) .
\end{aligned}
$$

Now we define

$$
\Sigma\left(\mathbf{x}_{2}, \mathbf{p}_{2}, \tau_{2} \mid \mathbf{x}_{1}, \mathbf{p}_{1}, \tau_{1}\right)=\iint d \mathbf{p} d \mathbf{p}^{\prime} g^{\prime}\left(\mathbf{x}_{1}, \mathbf{x}_{2}, \mathbf{p}, \mathbf{p}^{\prime}\right) K_{0}\left(\mathbf{x}_{2}, \mathbf{p}_{2}-\mathbf{p}^{\prime}, \tau_{2} \mid \mathbf{x}_{1}, \mathbf{p}_{1}+\mathbf{p}, \tau_{1}\right),
$$

such that

$$
K_{1}\left(\mathbf{x}_{b}, \mathbf{p}_{b}, t_{b} \mid \mathbf{x}_{a}, \mathbf{p}_{a}, t_{a}\right)=\int_{t_{a}}^{t_{b}} d \tau_{2} \int_{t_{a}}^{\tau_{2}} d \tau_{1} \iint d \mathbf{x}_{2} d \mathbf{p}_{2} \iint d \mathbf{x}_{1} d \mathbf{p}_{1} K_{0}(\mathbf{B} \mid \mathbf{2}) \Sigma(\mathbf{2} \mid \mathbf{1}) K_{0}(\mathbf{1} \mid \mathbf{A}),
$$


where we have introduced the following shorthand notation: $\mathbf{J}=\left\{\mathbf{x}_{j}, \mathbf{p}_{j}, \tau_{j}\right\}$. Exactly the same procedure can be repeated for the subsequent order terms in the perturbation series. The $n$th order in the series will have $2 n+1$ zeroth-order propagators $K_{0}$ and $n$ times $g^{\prime}$. One thus constructs $n$ selfenergies $\Sigma$, which leave $n+1$ zeroth-order propagators $K_{0}$ to connect all self-energies. Time ordering all the unordered time integrals will exactly cancel the $n$ ! term in the denominator. Such a structure immediately gives rise to a recurrence relation, from which we find the following Dyson integral equation for the propagator:

$$
\begin{aligned}
K_{w}(\mathbf{B} \mid \mathbf{A})= & K_{0}(\mathbf{B} \mid \mathbf{A})+\int_{t_{a}}^{t_{b}} d \tau_{2} \int_{t_{a}}^{\tau_{2}} d \tau_{1} \iint d \mathbf{x}_{2} d \mathbf{p}_{2} \\
& \times \iint d \mathbf{x}_{1} d \mathbf{p}_{1} K_{0}(\mathbf{B} \mid \mathbf{2}) \Sigma(\mathbf{2} \mid \mathbf{1}) K_{w}(\mathbf{1} \mid \mathbf{A}) .
\end{aligned}
$$

This constitutes the main result of the section. Before we finally turn our attention to the expression for the self-energy, we derive Eq. (2) from the Dyson equation above. Since $K_{0}$ is just the bare propagator associated with the bare action $S_{0}[\mathbf{x}, \xi]$, it satisfies the Wigner-Liouville equation [4]

$$
\begin{aligned}
& \left(\frac{\partial}{\partial t_{b}}+\frac{\mathbf{p}_{b}}{m} \cdot \nabla\right) K_{0}\left(\mathbf{x}_{b}, \mathbf{p}_{b}, t_{b} \mid \mathbf{x}_{a}, \mathbf{p}_{a}, t_{a}\right) \\
& \quad=\int d \mathbf{p}^{\prime} \Lambda\left(\mathbf{x}_{b}, \mathbf{p}_{b}-\mathbf{p}^{\prime}, t_{b}\right) K_{0}\left(\mathbf{x}_{b}, \mathbf{p}^{\prime}, t_{b} \mid \mathbf{x}_{a}, \mathbf{p}_{a}, t_{a}\right),
\end{aligned}
$$

which we denote as $\partial_{t_{b}} K_{0}(\mathbf{B} \mid \mathbf{A})+\hat{L}_{b} K_{0}(\mathbf{B} \mid \mathbf{A})=0$. Differentiating Eq. (8) with respect to the final time $t_{b}$ yields

$$
\begin{aligned}
\frac{\partial}{\partial t_{b}} K_{w}(\mathbf{B} \mid \mathbf{A}) \\
=\frac{\partial}{\partial t_{b}} K_{0}(\mathbf{B} \mid \mathbf{A})+\int_{t_{a}}^{t_{b}} d \tau_{2} \int_{t_{a}}^{\tau_{2}} d \tau_{1} \iint d \mathbf{x}_{2} d \mathbf{p}_{2} \\
\quad \times \iint d \mathbf{x}_{1} d \mathbf{p}_{1} \frac{\partial}{\partial t_{b}} K_{0}(\mathbf{B} \mid \mathbf{2}) \Sigma(\mathbf{2} \mid \mathbf{1}) K_{w}(\mathbf{1} \mid \mathbf{A}) \\
\quad+\int_{t_{a}}^{t_{b}} d \tau_{1} \iint d \mathbf{x}_{2} d \mathbf{p}_{2} \iint d \mathbf{x}_{1} d \mathbf{p}_{1} K_{0}\left(\mathbf{B} \mid \mathbf{x}_{2}, \mathbf{p}_{2}, t_{b}\right) \\
\quad \times \Sigma\left(\mathbf{x}_{2}, \mathbf{p}_{2}, t_{b} \mid \mathbf{1}\right) K_{w}(\mathbf{1} \mid \mathbf{A}) .
\end{aligned}
$$

In the last line, we now find a propagator where the initial and final times are equal. By definition of the propagator, this yields

$$
K_{0}\left(\mathbf{B} \mid \mathbf{x}_{2}, \mathbf{p}_{2}, t_{b}\right)=\delta\left(\mathbf{x}_{b}-\mathbf{x}_{2}\right) \delta\left(\mathbf{p}_{b}-\mathbf{p}_{2}\right) .
$$

If we furthermore introduce $\hat{L}_{b}$ and take it out of the integral, we find

$$
\begin{aligned}
& \frac{\partial}{\partial t_{b}} K_{w}(\mathbf{B} \mid \mathbf{A}) \\
& =-\hat{L}_{b}\left[K_{0}(\mathbf{B} \mid \mathbf{A})+\int_{t_{a}}^{t_{b}} d \tau_{2} \int_{t_{a}}^{\tau_{2}} d \tau_{1} \iint d \mathbf{x}_{2} d \mathbf{p}_{2}\right. \\
& \left.\quad \times \iint d \mathbf{x}_{1} d \mathbf{p}_{1} K_{0}(\mathbf{B} \mid \mathbf{2}) \Sigma(\mathbf{2} \mid \mathbf{1}) K_{w}(\mathbf{1} \mid \mathbf{A})\right] \\
& +\int_{t_{a}}^{t_{b}} d \tau_{1} \iint d \mathbf{x}_{1} d \mathbf{p}_{1} \Sigma(\mathbf{B} \mid \mathbf{1}) K_{w}(\mathbf{1} \mid \mathbf{A})
\end{aligned}
$$

The term between the square brackets is exactly the right-hand side of Eq. (8), such that we arrive at

$$
\begin{aligned}
& \frac{\partial}{\partial t_{b}} K_{w}(\mathbf{B} \mid \mathbf{A})+\hat{L}_{b} K_{w}(\mathbf{B} \mid \mathbf{A}) \\
& \quad=\int_{t_{a}}^{t_{b}} d \tau_{1} \iint d \mathbf{x}_{1} d \mathbf{p}_{1} \Sigma(\mathbf{B} \mid \mathbf{1}) K_{w}(\mathbf{1} \mid \mathbf{A}) .
\end{aligned}
$$

If we multiply the expression with the initial reduced Wigner function and integrate over $\left(\mathbf{x}_{a}, \mathbf{p}_{a}\right)$, we find the equation of motion for the reduced Wigner function,

$$
\frac{\partial}{\partial t_{b}} f(\mathbf{B})+\hat{L}_{b} f(\mathbf{B})=\int_{t_{a}}^{t_{b}} d \tau_{1} \iint d \mathbf{x}_{1} d \mathbf{p}_{1} \Sigma(\mathbf{B} \mid \mathbf{1}) f(\mathbf{1}) .
$$

At this point, it is safe to take the limit of $t_{a} \rightarrow-\infty$, such that the assumption of an initial product state of bath and particle was infinitely long ago. Defining the retarded self-energy as

$$
\Sigma_{R}(\mathbf{2} \mid \mathbf{1})=\Theta\left(t_{2}-t_{1}\right) \Sigma(\mathbf{2} \mid \mathbf{1}),
$$

and replacing $\hat{L}_{b}$ with its specific expression, we finally arrive at the quantum Liouville equation (2) announced in the Introduction:

$$
\begin{aligned}
& \left(\frac{\partial}{\partial t}+\frac{\mathbf{p}}{m} \cdot \nabla\right) f(\mathbf{x}, \mathbf{p}, t) \\
& \quad=\int d \mathbf{p}^{\prime} \Lambda\left(\mathbf{x}, \mathbf{p}-\mathbf{p}^{\prime}, t\right) f\left(\mathbf{x}, \mathbf{p}^{\prime}, t\right) \\
& \quad+\iiint d t^{\prime} d \mathbf{x}^{\prime} d \mathbf{p}^{\prime} \Sigma_{R}\left(\mathbf{x}, \mathbf{p}, t \mid \mathbf{x}^{\prime}, \mathbf{p}^{\prime}, t^{\prime}\right) f\left(\mathbf{x}^{\prime}, \mathbf{p}^{\prime}, t^{\prime}\right) .
\end{aligned}
$$

\section{INFLUENCE FUNCTIONAL AND SELF-ENERGY}

In this section, we show how to arrive at expression (3) or the retarded self-energy $\Sigma_{R}$ from its basic definition (7). To do so, we need the influence functional $[1,5]$ caused by the bath. A detailed derivation of the influence functional of a harmonic subsystem linearly coupled to the system was presented in Ref. [1]. Representing the bosonic bath operator in terms of the harmonic oscillator ladder operator directly turns the action associated with Hamiltonian (1) into the form discussed in [1]. Here we present a much shorter derivation, using coherent state Wigner functions [6] for the bath. The Weyl ordered Lagrangian associated with Hamiltonian (1) is

$$
\begin{aligned}
\mathcal{L}_{W}\left(\mathbf{x}, \dot{\mathbf{x}},\left\{b_{k}\right\},\left\{b_{k}^{*}\right\}\right) & \\
= & \frac{m \dot{\mathbf{x}}^{2}}{2}+\frac{1}{2} \sum_{k}\left(b_{k}^{*} i \hbar \frac{\partial b_{k}}{\partial t}-b_{k} i \hbar \frac{\partial b_{k}^{*}}{\partial t}\right)-V(\mathbf{x}, t) \\
& -\sum_{k} \hbar \omega_{k} b_{k}^{*} b_{k} \\
& -\sum_{k}\left(\gamma(\mathbf{k}) \exp (-i \mathbf{k} \cdot \mathbf{x}) b_{k}^{*}+\gamma^{*}(\mathbf{k}) \exp (+i \mathbf{k} \cdot \mathbf{x}) b_{k}\right) .
\end{aligned}
$$

Since the action is harmonic in the bath variables, its Wigner propagator is just a $\delta$ function along the classical trajectory $[1,6,7]$. Hence in complete analogy with the derivation in [1], we find the following expression for the influence 
functional:

$$
\begin{aligned}
\mathcal{F}[\mathbf{x}+\boldsymbol{\xi} / 2, \mathbf{x}-\boldsymbol{\xi} / 2]= & \exp \left[\frac{i}{\hbar} \sum_{k} \frac{4|\gamma(\mathbf{k})|^{2}}{\hbar} \int_{t_{a}}^{t_{b}} \int_{t_{a}}^{t} \sin \left[\mathbf{k} \cdot\left(\mathbf{x}_{s}-\mathbf{x}_{t}\right)\right] \sin \left(\frac{\mathbf{k} \cdot \boldsymbol{\xi}_{t}}{2}\right) \cos \left(\frac{\mathbf{k} \cdot \boldsymbol{\xi}_{s}}{2}\right) \sin \left(\omega_{k}[t-s]\right) \mathrm{d} s \mathrm{~d} t\right] \\
& \times \prod_{k}\left\{\iint d b_{k} d b_{k}^{*} \exp \left[\frac{2 \gamma(\mathbf{k})}{\hbar} b_{k}^{*} \int_{t_{a}}^{t_{b}} e^{i \omega_{k}\left(t-t_{a}\right)-i \mathbf{k} \cdot \mathbf{x}_{t}} \sin \left(\frac{\mathbf{k} \cdot \boldsymbol{\xi}_{t}}{2}\right) d t-\text { H.c. }\right] f_{k}\left(b_{k}, b_{k}^{*}\right)\right\} .
\end{aligned}
$$

Despite the classical trajectories which govern its dynamics, the influence functional (11) is intrinsically of a quantum-mechanical nature, because the initial Wigner distribution function $f_{k}\left(b_{k}, b_{k}^{*}\right)$ is bound to satisfy the uncertainty principle. Note that the first line in expression (11) is independent of the initial state of the bath. It represents the effective classical self-interaction, and it is time-symmetric. The time ordering $t_{a}<t_{b}$, as suggested by the notation, is not stringent. The factor on the second line, however, is state-dependent. It should be emphasized that a sharply defined (classical) initial distribution like $\delta^{2}\left(b_{k}-b_{0}\right)$ would result in a completely real influence phase. But such a distribution is not allowed, since it violates the uncertainty principle. Any quantum mechanically acceptable initial distribution results in a proper influence phase with an imaginary component. The imaginary part of the influence phase has the form of a stochastic potential acting on the system [1,5], inducing irreversibility onto the system, because it also propagates the uncertainty.

In the case of an initial thermal distribution with inverse temperature $\beta$, we have

$$
f_{k}\left(b_{k}, b_{k}^{*}\right)=\frac{2 \tanh \left(\beta \hbar \omega_{k} / 2\right)}{\pi} \exp \left[-2 \tanh \left(\beta \hbar \omega_{k} / 2\right)\left|b_{k}\right|^{2}\right] .
$$

The remaining Gaussian integral in expression (11) then results in the following expression for the influence phase:

$$
\begin{aligned}
\Phi[\mathbf{x}+\boldsymbol{\xi} / 2, \mathbf{x}-\boldsymbol{\xi} / 2]= & \sum_{k} \frac{4|\gamma(\mathbf{k})|^{2}}{\hbar} \int_{t_{a}}^{t_{b}} \int_{t_{a}}^{t} \sin \left[\mathbf{k} \cdot\left(\mathbf{x}_{s}-\mathbf{x}_{t}\right)\right] \sin \left(\frac{\mathbf{k} \cdot \boldsymbol{\xi}_{t}}{2}\right) \cos \left(\frac{\mathbf{k} \cdot \boldsymbol{\xi}_{s}}{2}\right) \sin \left(\omega_{k}[t-s]\right) d s d t \\
& +i \sum_{k} \frac{\operatorname{coth}\left(\frac{\beta \hbar \omega_{k}}{2}\right)}{2} \frac{4|\gamma(\mathbf{k})|^{2}}{\hbar} \int_{t_{a}}^{t_{b}} \int_{t_{a}}^{t_{b}} \cos \left[\mathbf{k} \cdot\left(\mathbf{x}_{s}-\mathbf{x}_{t}\right)\right] \sin \left(\frac{\mathbf{k} \cdot \boldsymbol{\xi}_{t}}{2}\right) \sin \left(\frac{\mathbf{k} \cdot \boldsymbol{\xi}_{s}}{2}\right) \cos \left(\omega_{k}[t-s]\right) d s d t .
\end{aligned}
$$

Time ordering of the last term removes the factor $1 / 2$ and brings the influence phase in the desired form (6), which allows us to extract $g\left(\mathbf{x}_{t}, \mathbf{x}_{s}, \boldsymbol{\xi}_{t}, \boldsymbol{\xi}_{s}\right)$ from the previous expression for $\Phi$. From definition (7), one arrives by straightforward algebra at the final expression (3) for the self-energy $\Sigma_{R}$, which we recapitulate here for convenience,

$$
\begin{aligned}
\Sigma_{R}\left(\mathbf{x}, \mathbf{p}, t \mid \mathbf{x}^{\prime}, \mathbf{p}^{\prime}, t^{\prime}\right)= & \Theta\left(t-t^{\prime}\right) \sum_{k} \frac{2|\gamma(\mathbf{k})|^{2}}{\hbar^{2}}\left[n_{B}\left(\omega_{k}\right) \cos \left[\mathbf{k} \cdot\left(\mathbf{x}-\mathbf{x}^{\prime}\right)-\omega_{k}\left(t-t^{\prime}\right)\right]+\left[n_{B}\left(\omega_{k}\right)+1\right] \cos \left[\mathbf{k} \cdot\left(\mathbf{x}-\mathbf{x}^{\prime}\right)+\omega_{k}\left(t-t^{\prime}\right)\right]\right] \\
& \times\left[K_{0}\left(\mathbf{x}, \mathbf{p}-\frac{\hbar \mathbf{k}}{2}, t \mid \mathbf{x}^{\prime}, \mathbf{p}^{\prime}+\frac{\hbar \mathbf{k}}{2}, t^{\prime}\right)-K_{0}\left(\mathbf{x}, \mathbf{p}+\frac{\hbar \mathbf{k}}{2}, t \mid \mathbf{x}^{\prime}, \mathbf{p}^{\prime}+\frac{\hbar \mathbf{k}}{2}, t^{\prime}\right)\right] .
\end{aligned}
$$

One readily identifies the scattering terms associated with emission and absorption of quanta from the reservoir. Moreover, note that, independent of the bare Hamiltonian of the system,

$$
\int d \mathbf{p} \Sigma_{R}\left(\mathbf{x}, \mathbf{p}, t \mid \mathbf{x}^{\prime}, \mathbf{p}^{\prime}, t^{\prime}\right)=\mathbf{0}
$$

which implies that the reduced distribution function satisfies the continuity equation.

\section{DISCUSSION}

As an important example, consider the bare system to be free, i.e., $H_{0}=\mathbf{p}^{2} / 2 m$. Since the problem is harmonic, its Wigner propagator is determined by the classical trajectory

$$
K_{0}\left(\mathbf{x}, \mathbf{p}, t \mid \mathbf{x}^{\prime}, \mathbf{p}^{\prime}, t^{\prime}\right)=\delta\left(\mathbf{p}-\mathbf{p}^{\prime}\right) \delta\left[\mathbf{x}-\left(\mathbf{x}^{\prime}+\frac{\mathbf{p}^{\prime}}{m}\left(t-t^{\prime}\right)\right)\right] .
$$

Note that for a bare free particle, both the bare and the total Hamiltonian of the system and bath are translational invariant. Let us therefore consider the initial reduced Wigner function of the system to be translational invariant too, i.e., $f_{0}(\mathbf{x}, \mathbf{p})=f(\mathbf{p})$. Under these conditions, the Liouville equation (2) for the reduced Wigner function becomes

$$
\begin{aligned}
\frac{\partial}{\partial t} f(\mathbf{p}, t)= & \sum_{k} \frac{2|\gamma(\mathbf{k})|^{2}}{\hbar^{2}} \int d s \Theta(t-s)\left[n_{B}\left(\omega_{k}\right) \cos \left(\left[\frac{(\mathbf{p}+\hbar \mathbf{k})^{2}}{2 m}-\frac{\mathbf{p}^{2}}{2 m}+\hbar \omega_{k}\right](t-s) / \hbar\right)+\left[n_{B}\left(\omega_{k}\right)+1\right]\right. \\
& \left.\times \cos \left(\left[\frac{(\mathbf{p}+\hbar \mathbf{k})^{2}}{2 m}-\frac{\mathbf{p}^{2}}{2 m}-\hbar \omega_{k}\right](t-s) / \hbar\right)\right] f(\mathbf{p}+\hbar \mathbf{k}, s)
\end{aligned}
$$




$$
\begin{aligned}
& -\sum_{k} \frac{2|\gamma(\mathbf{k})|^{2}}{\hbar^{2}} \int d s \Theta(t-s)\left[n_{B}\left(\omega_{k}\right) \cos \left(\left[\frac{(\mathbf{p}+\hbar \mathbf{k})^{2}}{2 m}-\frac{\mathbf{p}^{2}}{2 m}-\omega_{k}\right](t-s) / \hbar\right)+\left[n_{B}\left(\omega_{k}\right)+1\right]\right. \\
& \left.\times \cos \left(\left[\frac{(\mathbf{p}+\hbar \mathbf{k})^{2}}{2 m}-\frac{\mathbf{p}^{2}}{2 m}+\omega_{k}\right](t-s) / \hbar\right)\right] f(\mathbf{p}, s) .
\end{aligned}
$$

Consequently, any stationary solution of the Liouville equation should satisfy

$$
\begin{gathered}
\sum_{k} \frac{2 \pi|\gamma(\mathbf{k})|^{2}}{\hbar}\left[n_{B}\left(\omega_{k}\right) S_{+}\left(\mathbf{p}, \mathbf{k}, \omega_{k}\right)+\left[n_{B}\left(\omega_{k}\right)+1\right] S_{-}\left(\mathbf{p}, \mathbf{k}, \omega_{k}\right)\right] f(\mathbf{p}+\hbar \mathbf{k}) \\
=\sum_{k} \frac{2 \pi|\gamma(\mathbf{k})|^{2}}{\hbar}\left[n_{B}\left(\omega_{k}\right) S_{-}\left(\mathbf{p}, \mathbf{k}, \omega_{k}\right)+\left[n_{B}\left(\omega_{k}\right)+1\right] S_{+}\left(\mathbf{p}, \mathbf{k}, \omega_{k}\right)\right] f(\mathbf{p}),
\end{gathered}
$$

with

$$
S_{ \pm}\left(\mathbf{p}, \mathbf{k}, \omega_{k}\right)=\int \frac{d s}{\pi \hbar} \Theta(t-s) \cos \left(\left[\frac{(\mathbf{p}+\hbar \mathbf{k})^{2}}{2 m}-\frac{\mathbf{p}^{2}}{2 m} \pm \hbar \omega_{k}\right](t-s) / \hbar\right)=\delta\left(\frac{(\mathbf{p}+\hbar \mathbf{k})^{2}}{2 m}-\frac{\mathbf{p}^{2}}{2 m} \pm \hbar \omega_{k}\right) .
$$

Clearly the transition rates are now determined by Fermi's golden rule. Moreover, Eq. (14) now manifests detailed balance, from which we immediately find that

$$
\frac{f(\mathbf{p}+\hbar \mathbf{k})}{f(\mathbf{p})}=\frac{n_{B}\left(\frac{(\mathbf{p}+\hbar \mathbf{k})^{2}}{2 m}-\frac{\mathbf{p}^{2}}{2 m}\right)}{n_{B}\left(\frac{(\mathbf{p}+\hbar \mathbf{k})^{2}}{2 m}-\frac{\mathbf{p}^{2}}{2 m}\right)+1}=\exp \left[-\beta\left(\frac{(\mathbf{p}+\hbar \mathbf{k})^{2}}{2 m}-\frac{\mathbf{p}^{2}}{2 m}\right)\right] .
$$

This implies that the only stationary reduced Wigner function for the system is given by a Maxwell-Boltzmann distribution. Note that this result is independent of the strength of the coupling $\gamma$ and the nature of the bath $\omega_{k}$. With this in mind, we perturb the system by adding an external force such that

$$
H_{0}=\frac{\mathbf{p}^{2}}{2 m}-\mathbf{F} \cdot \mathbf{x} .
$$

Since the bare system Hamiltonian remains quadratic, we find

$$
K_{0}\left(\mathbf{x}, \mathbf{p}, t \mid \mathbf{x}^{\prime}, \mathbf{p}^{\prime}, t^{\prime}\right)=\delta\left\{\mathbf{p}-\left[\mathbf{p}^{\prime}+\mathbf{F}\left(t-t^{\prime}\right)\right]\right\} \delta\left[\mathbf{x}-\left(\mathbf{x}^{\prime}+\frac{\mathbf{p}^{\prime}}{m}\left(t-t^{\prime}\right)+\frac{\mathbf{F}}{2 m}\left(t-t^{\prime}\right)^{2}\right)\right] .
$$

If we again consider the initial distribution to be homogeneous, we arrive at the following Liouville equation for the perturbed system:

$$
\begin{aligned}
\left(\frac{\partial}{\partial t}+\mathbf{F} \cdot \nabla_{p}\right) f(\mathbf{p}, t)= & \sum_{k} \frac{2|\gamma(\mathbf{k})|^{2}}{\hbar^{2}} \int d s \Theta(s)\left[n_{B}\left(\omega_{k}\right) \cos \left(\Delta E_{+} s / \hbar-\frac{\mathbf{k} \cdot \mathbf{F}}{2 m} s^{2}\right)+\left[n_{B}\left(\omega_{k}\right)+1\right] \cos \left(\Delta E_{-} s / \hbar-\frac{\mathbf{k} \cdot \mathbf{F}}{2 m} s^{2}\right)\right] \\
& \times f(\mathbf{p}+\hbar \mathbf{k}-\mathbf{F} s, t-s)-\sum_{k} \frac{2|\gamma(\mathbf{k})|^{2}}{\hbar^{2}} \int d s \Theta(s)\left[n_{B}\left(\omega_{k}\right) \cos \left(\Delta E_{-} s / \hbar-\frac{\mathbf{k} \cdot \mathbf{F}}{2 m} s^{2}\right)\right. \\
& \left.+\left[n_{B}\left(\omega_{k}\right)+1\right] \cos \left(\Delta E_{+} s / \hbar-\frac{\mathbf{k} \cdot \mathbf{F}}{2 m} s^{2}\right)\right] f(\mathbf{p}-\mathbf{F} s, t-s)
\end{aligned}
$$

where we introduced $\Delta E_{ \pm}\left(\mathbf{p}, \mathbf{k}, \omega_{k}\right)=\frac{(\mathbf{p}+\hbar \mathbf{k})^{2}}{2 m}-\frac{\mathbf{p}^{2}}{2 m} \pm \hbar \omega_{k}$ for notational simplicity. The right-hand side of this equation is highly non-Markovian and difficult to treat. However, note that as a result of the bare propagator $K_{0}$ in the expression of the self-energy, the Wigner function should be evaluated at a time $s$ before the current time while also being displaced along the classical trajectory. In the absence of the bath, this would exactly yield the Wigner function at the current time. Hence under weak-coupling conditions, we have

$$
f(\mathbf{p}-\mathbf{F} s, t-s) \approx f(\mathbf{p}, t)+O\left[|\gamma(\mathbf{k})|^{2}\right], \quad f(\mathbf{p}+\hbar \mathbf{k}-\mathbf{F} s, t-s) \approx f(\mathbf{p}+\hbar \mathbf{k}, t)+O\left[|\gamma(\mathbf{k})|^{2}\right] .
$$

As the right-hand side of the equation of motion already contains a factor $|\gamma(\mathbf{k})|^{2}$, we can neglect the correction to the classical trajectory in weak coupling. This approximation results in the following Markovian Liouville equation:

$$
\begin{aligned}
\left(\frac{\partial}{\partial t}+\mathbf{F} \cdot \nabla_{p}\right) f(\mathbf{p}, t) \approx & \sum_{k} \frac{2 \pi|\gamma(\mathbf{k})|^{2}}{\hbar}\left[n_{B}\left(\omega_{k}\right) S_{+}\left(\mathbf{p}, \mathbf{k}, \omega_{k}\right)+\left[n_{B}\left(\omega_{k}\right)+1\right] S_{-}\left(\mathbf{p}, \mathbf{k}, \omega_{k}\right)\right] f(\mathbf{p}+\hbar \mathbf{k}, t) \\
& -\sum_{k} \frac{2 \pi|\gamma(\mathbf{k})|^{2}}{\hbar}\left[n_{B}\left(\omega_{k}\right) S_{-}\left(\mathbf{p}, \mathbf{k}, \omega_{k}\right)+\left[n_{B}\left(\omega_{k}\right)+1\right] S_{+}\left(\mathbf{p}, \mathbf{k}, \omega_{k}\right)\right] f(\mathbf{p}, t),
\end{aligned}
$$


where

$$
S_{ \pm}\left(\mathbf{p}, \mathbf{k}, \omega_{k}\right)=\int_{0}^{\infty} \frac{d \beta}{\pi} \cos \left(\beta \Delta E_{ \pm}-\frac{\hbar^{2} \mathbf{k} \cdot \mathbf{F}}{2 m} \beta^{2}\right) .
$$

It follows immediately that under linear-response conditions, i.e., when the perturbation is weak, the scattering again becomes

$$
\lim _{\mathbf{F} \rightarrow 0} S_{ \pm}\left(\mathbf{p}, \mathbf{k}, \omega_{k}\right)=\delta\left(\frac{(\mathbf{p}+\hbar \mathbf{k})^{2}}{2 m}-\frac{\mathbf{p}^{2}}{2 m} \pm \hbar \omega_{k}\right),
$$

and the reduced Liouville equation in the system simply becomes a classical Boltzmann equation where the scattering rates are given by Fermi's golden rule. Moreover, note that the force-dependent term in $S_{ \pm}$is quantum in nature as it scales with $\hbar^{2}$. In this respect, the reduction of the scattering amplitudes to Fermi's golden rule under the assumption of vanishingly small perturbation can also be interpreted as a truncation of the exact quantum result up to the classical result. Similarly one can, for an isolated system, truncate the Moyal bracket up to the Poisson bracket, which also results in a quantum correction of order $\hbar^{2}$ because the Poisson bracket does not incorporate any interference effects in the time evolution. In this context, this approximation is known as the truncated Wigner approximation [6]. The $\beta$ integral which determines the scattering amplitude can, in this case, be done exactly as it is just a complex Gaussian integral. Doing so, one finds that the resulting function tends to a $\delta$ function in a rather complicated way. On the one hand, depending on the sign of $\mathbf{k} \cdot \mathbf{F}$, the scattering amplitude oscillates rapidly. On the other hand, it decays monotonically. This oscillating behavior of the scattering amplitude again indicates the quantum nature of the scattering as it makes the scattering amplitude negative for some (nonclassical) transitions. This in turn allows the Wigner function to becomes negative.

Finally, note that, although a single scattering event does not have to preserve energy anymore, the expected energy difference is still zero as the first moment of $S_{ \pm}$with respect to $\Delta E_{ \pm}$vanishes

\section{CONCLUSION}

This work introduces the Wigner-Liouville equation for the reduced distribution function of a system coupled to a bath of harmonic oscillators. It shows how the retarded self-energy can be calculated from the influence functional of the reduced propagator. In general, the resulting Liouville equation is highly non-Markovian. How to deal with it is still a subject of current research. However, it was shown that under conditions of weak coupling, the time evolution approximately becomes Markovian. An additional assumption of linear response moreover results in Fermi's golden rule for the scattering rates. Alternatively, one can interpret Fermi's golden rule as the classically truncated version of the exact quantum amplitudes. Whereas the exact amplitudes include events which change the sign of the Wigner distribution, Fermi's golden rule does not. Finally, it was shown that, whenever the bare system is a free particle, the stationary distribution satisfies detailed balance conditions and is consequently given by a Maxwell-Boltzmann distribution.
[1] D. Sels, F. Brosens, and W. Magnus, Physica A 392, 326 (2013).

[2] A. O. Caldeira and A. J. Leggett, Physica A 121, 587 (1983).

[3] H. Kleinert, Nonequilibrium Quantum Statistics, in Path Integrals in Quantum Mechanics, Statistics, Polymer Physics, and Financial Markets, 5th ed. (World Scientific, Singapore, 2009), Chap. 18, Free online access at http://klnrt.de/b5.
[4] D. Sels, F. Brosens, and W. Magnus, Phys. Lett. A 376, 809 (2012).

[5] R. P. Feynman and F. L. Vernon, Jr., Ann. Phys. 24, 118 (1963).

[6] A. Polkovnikov, Ann. Phys. 325, 1790 (2010).

[7] H.-T. Elze, G. Gambarotta, and F. Vallone, J. Phys. Conf. Ser. 306, 012010 (2011); Int. J. Quant. Inf. 09 Suppl. 1, 203 (2011). 